\title{
On the Evolution of Virulent Zoonotic Viruses in Bats
}

\author{
Frans L. Roes ${ }^{1}$ \\ Received: 6 May 2020 / Accepted: 21 September 2020 / Published online: 16 October 2020 \\ c) Konrad Lorenz Institute for Evolution and Cognition Research 2020
}

\begin{abstract}
Ideas formulated by Paul Ewald about the "evolution of virulence" are used to explain why bats, more often than other mammals, are a reservoir of virulent viruses, and why many of these viruses severely affect other mammals, including humans, but are apparently less pathogenic for bats. Potential factors contributing to bat viruses often being zoonotic are briefly discussed.
\end{abstract}

Keywords Evolution of virulence $\cdot$ Hosts $\cdot$ Parasites $\cdot$ Reservoir of virulent viruses $\cdot$ Transmission mode $\cdot$ Zoonosis

\section{Introduction}

Bats are exceptional in several ways. They are the only flying mammals, many species have echolocation, they enjoy extraordinary longevity for their size, and the viruses they host are exceptional. Every life form investigated has been shown to host viruses (Calisher et al. 2006, p. 542). Several viruses hosted by bats, like henipaviruses, coronaviruses, filoviruses, and the rabies-causing lyssaviruses have been shown to be transmissible from bats to humans (Wynne and Wang 2013, p. 1). In other words, they are zoonotic. Another remarkable aspect is that many of the viruses coming from bats are highly pathogenic for other mammals (including humans), but apparently less pathogenic for the bats themselves.

The theory of Ewald (1993a, b), the "evolution of virulence," is used here to answer three related questions.

A. Why are some infectious diseases more harmful than others?

B. Why are bats, more than other mammals, a reservoir of virulent zoonotic diseases?

C. Why is it that many of the viruses that severely affect other mammals, including humans, are apparently less pathogenic for bats?

Frans L. Roes

froes@dds.nl

https://www.froes.dds.nl

1 Amsterdam, The Netherlands

\section{Why are Some Infectious Diseases More Harmful Than Others?}

What follows is a concise description of Paul Ewald's theory of the evolution of virulence (from Roes 2018, pp. 243-244):

While suffering from a bout of diarrhea in the late 1970s, Ewald speculated that his body was being manipulated by some parasite or "guest." The benefit to the pathogen causing the sickness was the potential transmission to other hosts. Much like the particulates expelled during coughing, diarrhea can be a means of distribution, for instance, when a sewer leaks into drinking water. The "transmission mode" or means of distribution of parasites to new hosts became the key variable in Ewald's theory.

If parasites depend on the movement of infected hosts to reach new hosts, they tend to be friendly to their current host, because they are interested in keeping this host healthy and mobile, so it can spread the parasite to new hosts. Somewhat benign host-guest relations are therefore expected when transmission to new hosts is impaired by illness.

Quite the contrary holds when new hosts can be infected, even if the host is not healthy. This may happen when parasites are able to survive for a long time independent of the host, and can "sit and wait" for new hosts to come around, as anthrax does. Or when parasites are transported to new hosts by a vector, for instance a mosquito, a syringe, or a sewer leaking into drinking water. In such cases the current host need not stay healthy and alive for the parasite to infect new hosts, so the parasite can evolve to deplete the 
resources of the current host while attempting to reproduce maximally. Unimpeded reproducing parasites engender sickness in the host. In some cases, parasites killing their hosts may even outreproduce less damaging variants. This is possible because increased replication may lead to a level of transmission into new hosts that exceeds the loss of transmission resulting from the host's death. So in these cases natural selection results in guests that exploit their hosts intensely. Microorganisms are often capable of producing several new generations within a very short time and, through the process of natural selection, can therefore quickly adapt to new environments. More specifically, parasites can quickly evolve to either being more or less virulent, depending on the opportunities to transmit to new hosts. Ewald and others scrutinized numerous infectious diseases, and found substantial empirical support for the idea that virulence varies with transmission opportunities.

To summarize Ewald's theory in one sentence: if disease of the current host does not impede infection of new hosts, then natural selection will favor the more virulent variants of the parasite, because these make more copies of themselves (and are therefore more damaging to hosts). Or, even more succinctly: the easier it is for a parasite to be transmitted from very sick hosts, the more virulent the parasite will be.

The flu viruses serve as good examples. They are very contagious, yet the more virulent strains are usually not very successful in infecting new hosts, since current hosts tend to stay at home and in bed. By contrast, individuals infected with milder variants often proceed with their daily routine, shaking hands and spreading cough droplets. So the milder variants have more reproductive success than the virulent ones.

It is a different story when infected hosts do not stay home, not even when they are gravely ill from the virus, and continue to infect new hosts, as happened during World War I in the trenches. Ewald (1991) argued that in the humid trenches, the tents, the overcrowded hospitals, and trains, the virulent variant evolved which later became known as the Spanish flu.

\section{Why are Bats, More Than Other Mammals, a Reservoir of Virulent Zoonotic Diseases?}

Bats harbor a significantly higher proportion of zoonotic viruses than all other mammalian orders (Olival et al. 2017, p. 646; disputed by Mollentze and Streicker 2020; see also Watson 2020). This is remarkable because, for instance, there are about twice as many species of rodents as there are species of bats, and rodents are more closely related to humans than bats are. Why are bats a reservoir of virulent viruses?

Many bat species are gregarious, some living in dense aggregations. Colonies can reach densities of 3000 bats per square meter, in populations of up to a million individuals per roost (Luis et al. 2013, p. 2). The theory of virulence implicates the close quarters of bats as a factor favoring increased virulence because bats roost so closely to each other that they can transmit infections to other bats even if they are immobilized by illness. A more virulent variant, making more copies of itself, will therefore spread. Note that it is not closeness per se that favors virulent diseases, but closeness favors transmission from animals that are not mobile.

To summarize: the extreme closeness of bats in many roosting sites allows the transmission of viruses from very sick hosts, favoring the more virulent variants in the population.

Why are many bat viruses also zoonotic? Several characteristics of bats seem to facilitate transmission to other host species. Bats are the only mammals with the capability of powered flight. This enables them to have a longer radius of action compared to terrestrial mammals and to have more direct or indirect contact with other animal species at different geographical locations. The mobility of bats probably allows bat viruses to be dispersed to humans and other mammals.

Whereas rodent species typically do not share communal nesting sites, roosting sites of bats can house diverse assemblages of multiple bat species (Luis et al. 2013, p. 3). This also may favor zoonosis. In the words of Ewald (pers. comm.): "The important point here is that multispecies populations may favor infection mechanisms that are not species specific and may thus allow for more frequent transmission across species including zoonotic transmission to humans."

Finally, bats enjoy remarkable longevity for their body size. Some insectivorous bats can live up to 35 years (Wang et al. 2011, p. 650). Persistent infections may allow for prolonged release of viruses and thus greater exposure of humans (or other species) to the viruses.

\section{Why is it that Viruses that Severely Affect Other Mammals, Including Humans, are Apparently Less Pathogenic for Bats?}

With the exception of the lyssaviruses (such as rabies), bats generally seem to harbor viruses with no clinical signs of disease; but while bats have developed the ability to coexist with many different viruses, some of these viruses have 
proved to be highly lethal in other mammalian hosts (Wynne and Wang 2013, p. 3). Why?

The earliest known bat fossil dates to 52.5 million years ago (Jepsen 1966), so bats and viruses have coevolved for a long time. Assuming the reasoning above is correct, then an "arms race" between bats and viruses has been going on in that period. On the one hand, presumably caused by bats roosting closely to each other, the viruses have continuously evolved to be more virulent. On the other hand, many species of bats have maintained, for some apparently important reason, gregarious aggregations. An inevitable consequence would have been that natural selection, generation after generation, selected bats that were somehow able to live and reproduce despite the virulent viruses. Wynne and Wang (2013, p. 3) write: "Accelerated evolution of innate immune genes may be a direct consequence of prolonged viral exposure, and therefore reflects the evolutionary adaptations that have led to the superior antiviral phenotype bats possess."

Perhaps flight provided a selective force for coexistence with viral parasites through a daily cycle that elevates metabolism and body temperature, analogous to the febrile response in other mammals (O'Shea et al. 2014, p. 741). This temperature argument could allow bats to control virulent pathogens more fully than in other species without this defense.

If gregariousness is associated with virulent viruses, then why didn't bats evolve to be more solitary, or to at least keep some social distance from other bats? I speculate that once grounded, bats are rather helpless creatures, indeed easy prey. But with their flight and echolocation they can exploit environments like caves accessed with difficulty by potential predators.

Here the vulnerable creatures are relatively safe, share body heat, can hibernate and reproduce-all in the company of virulent viruses.

Acknowledgments For their comments on previous drafts of this article, I would like to thank Hamilton McMillan, Jan Albert Smit, and in particular Paul Ewald.

\section{References}

Calisher CH, Childs JE, Field HE, Holmes KV, Schountz T (2006) Bats: important reservoir hosts of emerging viruses. Clin Microbiol Rev 19:531-545. https://doi.org/10.1128/CMR.00017-06

Ewald PW (1991) Transmission modes and the evolution of virulence. With special reference to cholera, influenza, and AIDS. Hum Nat. 2(1):1-30. https://doi.org/10.1007/BF02692179

Ewald PW (1993a) The evolution of virulence. Sci Am 268(4):86-93. https://doi.org/10.1038/scientificamerican0493-86

Ewald PW (1993b) Evolution of infectious disease. Oxford University Press, Oxford

Jepsen GL (1966) Early eocene bat from Wyoming. Science 154:13331339. https://doi.org/10.1126/science.154.3754.1333

Luis AD, Hayman DTS, O'Shea TJ, Cryan PM, Gilbert AT, Pulliam JRC et al (2013) A comparison of bats and rodents as reservoirs of zoonotic viruses: are bats special? Proc R Soc B 280:20122753. https://doi.org/10.1098/rspb.2012.2753

Mollentze N, Streicker DG (2020) Viral zoonotic risk is homogenous among taxonomic orders of mammalian and avian reservoir hosts. Proc Natl Acad Sci USA. https://doi.org/10.1073/pnas.19191 76117

Olival KJ, Parviez R, Hosseini PR, Zambrana-Torrelio C, Ross N, Bogich TL, Daszak P (2017) Host and viral traits predict zoonotic spillover from mammals. Nature 546:646-650. https://doi. org/10.1038/nature22975

O'Shea TJ, Cryan PM, Cunningham AA, Fooks AR, Hayman DTS, Luis AD et al (2014) Bat flight and zoonotic viruses. Emerg Infect Dis 20(5):741-745. https://doi.org/10.3201/eid2005.130539

Roes FL (2018) The curious case of the Spanish flu. Biol Theory 13:243-245. https://doi.org/10.1007/s13752-018-0307-9

Wang LF, Walker PJ, Poon LL (2011) Mass extinctions, biodiversity and mitochondrial function: are bats 'special' as reservoirs for emerging viruses? Curr Opin Virol 1:649-657. https://doi. org/10.1016/j.coviro.2011.10.013

Watson C (2020) Bats are a key source of human viruses-but they're not special. Nature. https://doi.org/10.1038/d41586-020-01096-Z

Wynne JW, Wang LF (2013) Bats and viruses: friend or foe? PLoS Pathog 9(10):1-4. https://doi.org/10.1371/journal.ppat.1003651

Publisher's Note Springer Nature remains neutral with regard to jurisdictional claims in published maps and institutional affiliations. 